\title{
Experiencias de formación en extensión universitaria. Aportes y desafíos institucionales
}

\author{
Leticia Núñez \\ leticiasoledadnz@gmail.com
}

\section{Fernanda Américo}

famerico@cup.edu.uy

Johanna Arias

jarias@cup.edu.uy

\section{Georgina Thevenet}

georginatr89@gmail.com

Centro Universitario Regional Litoral Norte. Universidad de la República, Uruguay.
A 100 años de la Reforma Universitaria de 1918 / Intervenciones

RECEPCIÓN: 29/06/17

ACEPTACIÓN FINAL: 24/10/17

\section{Resumen}

En un contexto institucional de curricularización de la extensión desde una concepción de integralidad de las funciones universitarias y de la formación, se desarrollan estrategias pedagógicas a través de experiencias de formación en extensión y de vínculo universidad-sociedad. En este artículo se presentan dos experiencias de formación: por un lado, el Seminario de Formación en Extensión Universitaria y, por otro, el Curso Taller de Procesos Colectivos, Asociativismo y Cooperativismo. La primera experiencia es llevada a cabo por las Unidades de Extensión de las Sedes Salto y Paysandú, y la segunda, por la Unidad de Extensión de la Sede Salto, pertenecientes ambas al Centro Universitario Regional Litoral Norte (CENUR LN), Universidad de la República (UdelaR), Uruguay.

\section{Palabras clave}

- Formación en extensión

- Curricularización

- Integralidad

\section{Resumo}

Em um contexto institucional de curricularização da extensão a partir de uma concepção de integração de funções universitários e formação integral, estratégias educativas são desenvolvidas por meio de experiências de formação em extensão e ligação universidade-sociedade. Este artigo descreve duas experiências de formação apresentados, por um lado, o Seminário de Formação em Extensão Universitária, e em segundo lugar, a Oficina Processo Coletivo, Cooperativas e Curso Cooperativo. O primeiro experimento é conduzido pelas Unidades de Extensão Sede Salto e Paysandú, e o segundo pela Unidade de Extensão Sede Salto, pertencente ao Centro Acadêmico Regional Litoral Norte (CENUR LN), Universidade da República ( UdelaR), Uruguai.

Palavras-chave

- Formação em extensão

- Curricularização

- Integração

\section{Para citación de este artículo}

Núñez, L.; Américo, F., Arias, J. y Thevenet, G. (2017). Experiencias de formación en extensión universitaria. Aportes y desafíos institucionales. Revista + E versión en línea, 7(7), 234-243 Santa Fe, Argentina: Ediciones UNL. 
Breve reseña institucional de las prácticas integrales y curricularización de la extensión en la Universidad

\section{de la República}

En el contexto de la Segunda Reforma Universitaria de la Universidad de la República (UdelaR), podemos identificar una serie de ideas fuerza que condujeron a un proceso inicial de reforma y que influyeron directamente en el desarrollo de las propuestas pedagógicas que aquí se presentan.

El proceso de descentralización que la UdelaR llevó adelante en los últimos tiempos define la creación de Centros Universitarios Regionales (CENUR) en distintas zonas del país con el fin de desarrollar en cada uno de ellos estructuras académicas y administrativas, propuestas de formación desde los distintos servicios universitarios, así como la creación de nuevas ofertas de enseñanza terciaria. En este sentido, el CENUR Litoral Norte requiere la coordinación de las sedes universitarias presentes en Salto, Paysandú, Artigas y Río Negro.

Los conceptos de integralidad e interdisciplina y la definición político-académica de la curricularización de la extensión han sido también ideas fuerza de este proceso. Para materializarlas y ponerlas en práctica se han generado dispositivos pedagógicos tales como propuestas de cursos para la formación en extensión universitaria desde sus aspectos teóricos y metodológicos, destinados a estudiantes, docentes, egresados y actores sociales extrauniversitarios, y Espacios de Formación Integral en UdelaR:

"dispositivos pedagógicos flexibles que se conforman a partir de múltiples experiencias educativas en diálogo con la sociedad, asumiendo diferentes formas de reconocimiento curricular.

Buscan ser lugares donde el estudiante desarrolle prácticas integrales desde su ingreso a la Universidad, articulando las tres funciones universitarias". (2010:10)

Esta coyuntura política y de reformas pretendió revitalizar el valor inspirador del ideal latinoamericano de Universidad, aproximándolo a las condiciones sociales, políticas, económicas y culturales de nuestro tiempo y a la incorporación efectiva de la extensión al conjunto de actividades definitorias de nuestra institución mediante la curricularización de la extensión.

En los últimos años se ha instalado en la UdelaR el desafío de identificar los formatos pedagógicos e institucionales adecuados y pertinentes que permitan una formación profesional ajustada a la sociedad actual. En ese contexto se han esbozado propuestas que refieren a la integralidad de las funciones universitarias (investigación, extensión, enseñanza) como un punto básico y fundamental de la formación de grado de las carreras que ofrece la Universidad. La ordenanza de grado de la UdelaR considera clave: "incorporar en los planes de estudios los cursos optativos o electivos, la posibilidad de cursar unidades curriculares en otras carreras, la incorporación de prácticas de extensión y la participación en instancias a donde se integren funciones y disciplinas". (2011:5)

Por su parte, la Comisión Sectorial de Extensión y Actividades en el Medio (CSEAM), en su definición de extensión, la cual fue aprobada por el Consejo Directivo Central en el año 2009, sostiene que la extensión e investigación deberán ser parte de la metodología de enseñanza universitaria integral, en contacto directo con la realidad social.

En el marco del Plan Estratégico de la Universidad (PLEDUR, 2005) se da impulso al desarrollo de programas integrales, tomando en cuenta la experiencia acumulada al respecto de diversos equipos docentes de la universidad. En la región, como parte del Programa Regional de Enseñanza Terciaria (PRET) del CENUR Litoral Norte, las Unidades de Extensión de las Sedes Paysandú y Salto proponen llevar adelante una iniciativa que coordinara acciones y estrategias temáticas y territoriales, tomando a la integralidad como una práctica educativa que articula las funciones, disciplinas y saberes en territorio, a partir de una co-construcción de conocimientos basada en el análisis crítico, que permite aprender haciendo junto a los actores sociales involucrados en las experiencias. De esta manera y en consonancia con la política académica prorrectoral, se creó el Programa Integral Temático (PIT) correspondiente a los años 2013 a 2015 articular el quehacer universitario en el territorio y fortalecer el vínculo universidadsociedad. Como producto de un proceso de construcción colectiva entre docentes de distintos servicios universitarios y de las Unidades de Extensión de ambas sedes, surgieron las temáticas "Educación y comunidad" como ejes centrales del Programa, cuyo objetivo fue:

"Aportar en el marco de las políticas universitarias de renovación de la enseñanza, para la creación de un espacio que propicie el desarrollo de estrategias pedagógicas que promuevan la enseñanza activa, la interdisciplina y la integración de funciones universitarias, conjuntamente con los actores sociales en el área de influencia del CENUR Litoral Norte". (Apezteguía et al., 2013:3) 
EI PIT se constituyó sobre tres componentes interrelacionados: los Espacios de Formación Integral (EFI), el Seminario de Formación en Extensión y el Tiempo Integral de Campo. Con posterioridad al cierre de este programa, en el año 2015, el Seminario continúa desarrollándose como estrategia de curricularización de la extensión en el marco de distintas propuestas pedagógicas que llevan adelante ambas sedes.

\section{Experiencia del Seminario de Formación en Extensión Universitaria}

El Seminario ha sido el ámbito de formación transversal de todos los estudiantes y docentes que participaron de distintas experiencias del PIT. Actualmente, se enmarca en las actividades de promoción de la Extensión y las Prácticas Integrales que desarrollan las Unidades de Extensión del CENUR LN. En la Sede Salto, el Seminario es un componente del Programa Integral de la Cuenca del Arroyo San Antonio (PICASA 2016) que desarrollan docentes y estudiantes de dicha sede de la UdelaR. En la Sede Paysandú, forma parte de las ofertas de formación, abierta a todos los estudiantes de las distintas carreras que ofrece la sede, habiéndose curricularizado en el marco del Espacio de Formación Integral en Salud Comunitaria. El desarrollo del Seminario está a cargo de los equipos docentes de las Unidades de Extensión de las Sedes Salto y Paysandú y se realiza en dos ediciones semestrales, una en cada sede universitaria. Participan de él estudiantes y docentes de las áreas de salud, social y artística, y científico-tecnológica de distintos niveles de formación. En sus objetivos se plantea:

i) Sensibilizar a los/as estudiantes en la concepción de extensión universitaria y prácticas integrales.

ii) Compartir herramientas de extensión que aporten a las prácticas realizadas por los/as estudiantes en el medio.

iii) Generar un espacio de intercambio entre estudiantes y docentes en el cual se problematice la intervención de la Universidad en el medio.

Se trabaja bajo la modalidad de taller, entendido como metodología y no como técnica, lo cual permite el ordenamiento de las actividades en territorio y evita que se caiga en la espontaneidad y en el voluntarismo. En este sentido, se toma el aporte de Cano, quien entiende al espacio de taller desde una perspectiva de la educación popular, y lo define como:

"un dispositivo de trabajo con grupos, que es limitado en el tiempo y se realiza con determinados objetivos particulares, permitiendo la activación de un proceso pedagógico sustentado en la integración de teoría y práctica, el protagonismo de los participantes, el diálogo de saberes, y la producción colectiva de aprendizajes, operando una transformación en las personas participantes y en la situación de partida". (2012:33)

Los contenidos abordados en el seminario se organizan en las siguientes unidades:

- Unidad 1: conceptualización de actividad en el medio, práctica preprofesional y extensión universitaria. Recorrido histórico de la extensión universitaria. Modelos de extensión universitaria en la UdelaR. Presentación de experiencias sobre intervenciones de la Universidad en el medio.

- Unidad 2: herramientas de diagnóstico participativo. ¿Por qué participativo? ¿Cómo? Entrevista y grupo de discusión. Metodologías que promueven la participación. Mapeo de actores. Mapeo de problemas. Observación y cuaderno de campo.

- Unidad 3: herramientas de planificación participativa. Necesidades y problemas. Ordenamiento de problemas. Árbol de problemas. Identificación de áreas de acción. Actividades, valoración y análisis de alternativas. Evaluación. ¿Qué evaluar? ¿Cómo? ¿Para qué? - Unidad 4: reflexionar sobre nuestras prácticas. Aportes de la Investigación Acción Participativa. Aproximación a la sistematización de experiencias.

Luego de las instancias áulicas de formación, los estudiantes y docentes atraviesan por un tiempo de trabajo a campo, de acercamiento territorial y a la comunidad, adonde se van incorporando aspectos relacionados con la teoría a la vez que se establece un tiempo de reflexión práctica.

En la propuesta de espacio de formación integral que se vincula a este Seminario en la Sede Paysandú se trabaja junto a profesores y estudiantes de ciclo básico liceal en una institución educativa inserta en la ciudad. La línea temática abordada en este contexto es la salud comunitaria. En la Sede Salto se trabaja con la población que reside en el territorio delimitado por la cuenca del arroyo San Antonio, mayoritariamente en el medio rural y en algunos centros poblados.

La propuesta del Seminario se configura como primer antecedente curricular de formación en extensión en la región y marca el comienzo de un trabajo conjunto entre las sedes universitarias. Se presenta como estrategia pedagógica que contribuye a la formación del estudiante universitario y el quehacer docente desde una concepción de integralidad. Desde el equipo docente se lo considera como un espacio que promueve el encuentro de estudiantes de diversas disciplinas, al tiempo que propicia la identificación de aspectos comunes y disímiles de las prácticas desarrolladas y la co-construcción de conocimiento. Se busca que los estudiantes incorporen herramientas para el trabajo en la comunidad y diversas estrategias para la autogestión de sus propios conocimientos a partir de la construcción de un vínculo con los actores sociales con los que trabajan. 


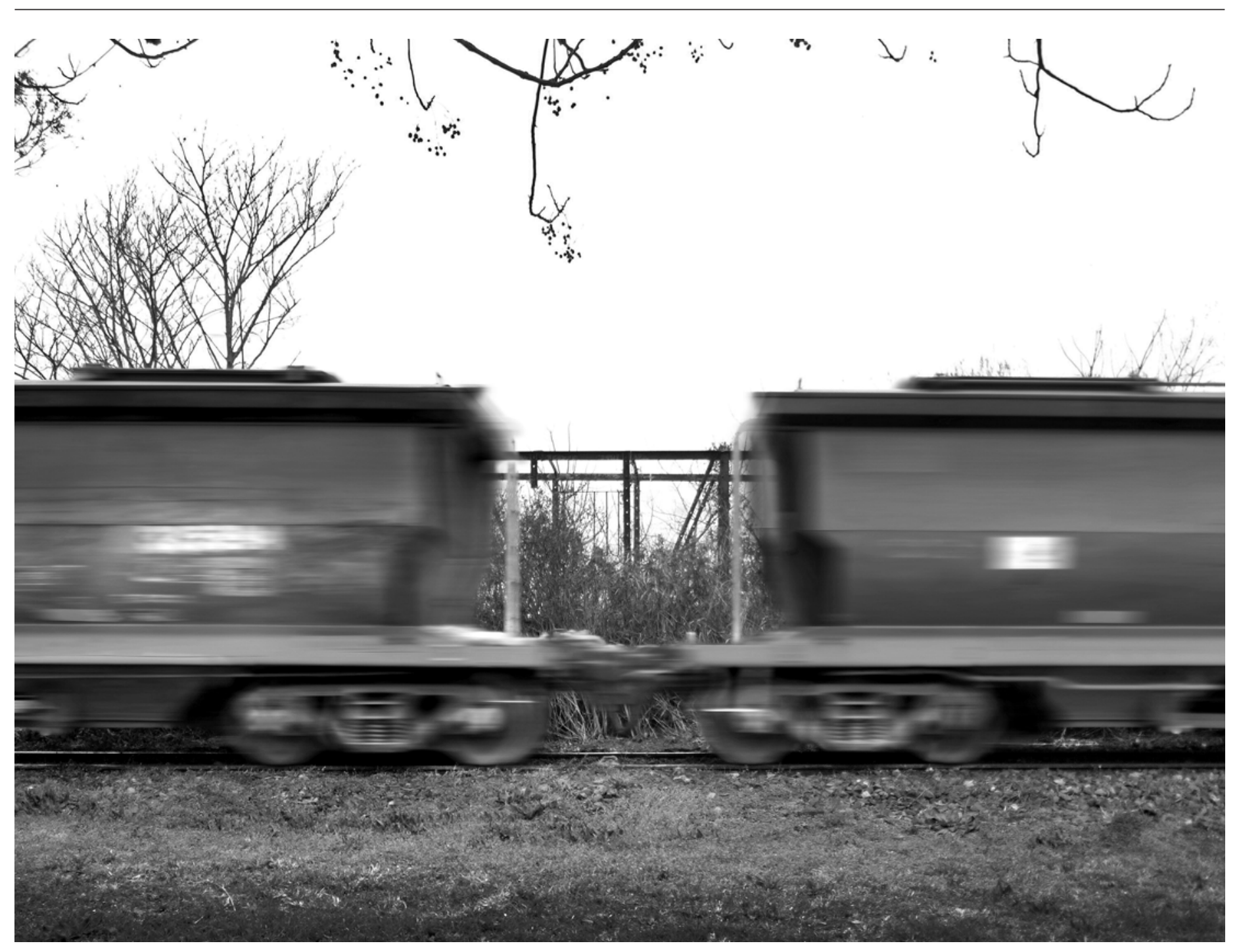

(C) Oscar Dechiara

\section{6}

Se busca que los estudiantes incorporen herramientas para el trabajo en la comunidad y diversas estrategias para la autogestión de sus propios conocimientos 


\section{Reflexiones y potencias de la experiencia}

Algunos elementos que hacen a la reflexión y análisis de esta experiencia de formación tienen que ver con la planificación dialógica, la dialéctica teoría-praxis y la interdisciplina. La planificación del Seminario se caracteriza por ser dialógica, dado que la misma incluye un proceso de acción-reflexión-acción de la práctica docente a través del análisis crítico grupal, donde el equipo docente analiza los contenidos y busca generar nuevas estrategias de tratamiento y abordaje de los mismos. La evaluación es un proceso destacable dentro de la planificación e involucra a docentes y estudiantes.

Destacamos las palabras de Luckesi sobre la importancia de la evaluación:

"La evaluación atraviesa el acto de planificar y de ejecutar; por eso contribuye en todo curso de la acción planificada. La evaluación no sólo en la identificación de la perspectiva político-social, como también en la selección de medios alternativos y en la ejecución del proyecto, sin perder de vista su construcción. O sea, la evaluación, como crítica de curso de la acción, es una herramienta necesaria para el ser humano en el proceso de construcción de los resultados que planificó producir, así como lo es el redimensionamiento de la dirección la acción. La evaluación es una herramienta de la cual el ser humano no puede librarse. Ella es parte de su modo de actuar y por eso, es necesario que sea usada de la mejor manera posible". (1974:118)

La planificación en un curso de formación en extensión se considera como una acción que contribuye a la búsqueda continua de nuevas formas de enseñar, de reconfigurar abordajes desde una dinámica de retroalimentación: docente-docente, docente-estudiante, estudiante-sociedad, docente-sociedad, docente-estudiante-sociedad. De esta manera, la planificación se jerarquiza ante el diálogo y la intención de perseguir el acto crítico de planificar. Cada edición del Seminario ha estado sujeta a los contenidos que se expresan en el programa, pero en la dinámica de planificar se presenta el desafío que impone no solo el hacer extensión sino el aprender a construir aprendizajes desde ella. Respecto de la dialéctica teoría-praxis, el Seminario debe conjugar lo teórico en una práctica situada que redunde luego en la teorización de la misma. En este sentido, cuidar el equilibrio del trabajo intrauniversitario y territorial se vuelve necesario, evitando así procesos endogámicos donde la extensión se deposite en un lugar meramente teórico y de abstracción.

Cabe mencionar que las Unidades de Extensión de las sedes universitarias insertas en el interior del país son transversales a la estructura académica y oferta educativa que en ellas se ofrece, por lo tanto, la interacción disciplinar y el trabajo interdisciplinar suelen surgir con cierta naturalidad o, al menos, pueden valerse de un terreno propicio para que suceda. Aun así, las disciplinas como compartimentos estancos, la objetividad y las concepciones positivistas de la ciencia están presentes siempre con fuerza. Najmanovich expresa algunos aspectos que hacen al concepto de disciplina:

"es preciso evocar los diversos significados de este término (...) el que atañe al discurso respecto de un área de conocimiento y el que está implicado en el acto de disciplinar, entendido como poner orden, corregir e incluso exigir obediencia (...) Una disciplina, entendida como área cognitiva, implica poner orden, organizar un discurso respecto de lo que se ha dado en llamar 'su objeto', recortar un área de pertenencia y construir sus herramientas de abordaje. Es más, las disciplinas no existen en abstracto, sino a través de la acción humana en el seno de una cultura y en un espacio tiempo determinado. Por lo tanto, este proceso tiene lugar en el seno de instituciones: las comunidades científicas. Estas establecen sus formas de comunicación, de validación, de relación tanto interna como con el contexto". (1998:1)

El equipo docente del Seminario de formación en extensión que es, en principio, multidisciplinar, hace un proceso de construcción colectiva conceptual y metodológica común de la propuesta pedagógica y logra transitar la interdisciplina en procura, a partir de las distintas disciplinas, desarrollar una propuesta desde la diversidad, donde entren en juego perspectivas teóricas y metodológicas no dogmáticas, no acabadas, no unívocas. Ello desde un enfoque complejo y en continua controversia, aportando algunos cuerpos conceptuales y herramientas que permitan la construcción e incorporación de nuevos conocimientos situados en prácticas concretas.

Así como el equipo docente del Seminario se construye de una manera interdisicplinar, los estudiantes que participan en él parten de una acción multidisciplinar que busca horizontes interdisciplinares, que en ocasiones logran revelarse. Estudiantes de distintas carreras y grados de avance en su formación conforman equipos de trabajo que tienen por delante el ejercicio de constituirse como tales en primera instancia, conocer y comprender desde perspectivas de análisis diversas, planificar y ensayar abordajes interdisciplinarios. 
Experiencias del curso taller de procesos colectivos, asociativismo y cooperativismo

Este curso es un espacio de formación que surgió en 2012 a partir de un acuerdo realizado entre la Sede Salto y la Unidad de Estudios Cooperativos del Servicio Central de Extensión y Actividades en el Medio (SCEAM) a los efectos de dar respuesta a una demanda del sector asociativo regional, en acuerdo con la Mesa Intercooperativa de Salto. Esta Mesa es una asociación civil que nuclea diversas modalidades de cooperativas con el fin de promover los valores y principios cooperativos en el medio, mediante la presentación de proyectos financiados por instituciones del sector cooperativo, realización de diferentes cursos de información y formación dirigidos a los cooperativistas consolidados o en proceso de conformación de una cooperativa. En 2012, precisamente entre febrero y marzo, se efectuó un llamado para un grado 1 con 10 horas semanales para desempeñar la función docente dentro del curso con la intención manifiesta de extenderla a 20 horas. La titular seleccionada se desempeñó como docente responsable desde mayo de dicho año.

Del equipo docente en ese año, además del grado 1 señalado, han participado integrantes de la Unidad de Estudios Cooperativos de Montevideo, quienes durante 2012 y 2013 estuvieron acompañando activamente en el diseño, planificación y ejecución del curso, además de aportar recursos materiales y financieros para el mismo. En los años siguientes, 2014 y 2015, su participación se redujo a instancias puntuales, con contribuciones en y con clases además de componer "mesas de debates" (presentación e intercambio de experiencias). El objetivo que tuvo la Unidad de Estudios Cooperativos fue la conformación y consolidación de un equipo docente local que pudiera encargarse del diseño y ejecución del curso.

Actualmente, el curso depende institucionalmente de la Unidad de Extensión del Centro Universitario Salto y es llevado a cabo por el grado 1 como responsable del mismo, con 10 horas semanales, recurso que se mantiene desde 2012, en colaboración con un docente grado 2 de la Unidad de Extensión de Salto.

Desde ese 2012 a la actualidad, se ha implementado desde la Unidad de Extensión de la Sede Salto este curso optativo para algunas carreras universitarias, donde la participación de estudiantes es bien disímil, pues tenemos desde carreras universitarias, tanto estudiantes como egresados, docentes, educadores, cooperativistas y trabajadores vinculados al sistema asociativo-cooperativo. El plazo de duración del curso es de abril a septiembre de cada año.

Durante estos años del cursado, se ha apostado a intercambiar y reflexionar colectivamente sobre la importancia de brindar este tipo de cursos dentro de la academia, pues la participación de actores es diversa; los estudiantes pertenecen no solo a la Universidad sino también al sector cooperativo-colectivo. Esta diversidad de actores es lo que nos permite un enriquecimiento por medio del intercambio colectivo de saberes, experiencias y la posibilidad de producir conocimiento a través de la realización de trabajos de investigación, de intervención o de extensión que los estudiantes en grupos multidisciplinarios deben desarrollar como uno de los requisitos fundamentales del curso. En cada edición, los docentes se plantean objetivos referentes a la promoción del conocimiento, estudio y reflexión sobre el cooperativismo, asociativismo y la economía social y solidaria en el Uruguay desde el abordaje universitario; generar un espacio de formación en el que estudiantes, docentes y trabajadores se vean desafiados por la temática y la construcción de un abordaje integral (enseñanzaextensión-investigación) e interdisciplinario; desarrollar los principales conceptos teórico-prácticos sobre el cooperativismo, asociativismo y la economía social y solidaria; promover debate y nuevas búsquedas a partir del recorte temático seleccionado en los espacios de trabajo en grupos multidisciplinarios; posibilitar, a través del trabajo en grupos, el acercamiento a prácticas de investigación, docencia y/o extensión procurando una aproximación a la temática cooperativa y asociativa y, por último pero no menos relevante, propiciar la práctica colectiva e interdisciplinaria/multidisciplinaria por medio de una reflexión crítica sobre los procesos asociativos, incorporando nuevos abordajes sobre lo "colectivo". Para cumplir con estos objetivos, es fundamental que exista una apuesta permanente en el compromiso en todo el proceso por parte de los estudiantes y de los docentes en cada edición.

Desde instancias generales y con una modalidad altamente participativa, el curso abarca los asuntos propios del tema: caracterización del cooperativismo en el Uruguay; evolución de los procesos sociohistóricos del sector cooperativo-asociativo; aspectos jurídicos del sector, economía social y solidaria, aportes de la educación popular al cooperativismo, metodologías participativas, entre otros.

Contempla abordajes metodológicos a través de salidas de campo, trabajo en equipos multidisciplinarios y realización de informes de investigación y/o extensión, "mesas de debate" (este espacio articulado en consulta con los participantes del curso a partir del interés general sobre un abordaje determinado) y se culmina con un trabajo de investigación, intervención o extensión, elaborado por los participantes. 
La totalidad de participantes en estos seis años fue de 257 y ellos provinieron desde diferentes ámbitos académicos (como estudiantes y egresados de la Universidad y de institutos de formación docente); profesionales (que se desempeñan en el sector cooperativo y colectivo) y cooperativistas (de distintas modalidades de cooperativas).

Luego de finalizada cada edición, los docentes junto a los estudiantes de cada edición se replantean algunas cuestiones metodológicas, a los efectos de mejorar, perfeccionar y consolidar el curso. Luego de esas instancias (individual y colectiva), desde el cuerpo docente se arma un documento a los efectos de que queden allí expresiones, aportes, o simplemente cuestiones realizadas por los estudiantes que son considerados a la hora de implementar otra nueva edición. En esas evaluaciones se han obtenido resultados interesantes, con aportes sustantivos, desde el punto de vista del conocimiento general del tema, reflexiones pertinentes sobre la realidad y actualidad del sector asociativo en el medio local e incluso en torno a la relación cooperativismo, universidad y sector asociativo-cooperativo.

Los trabajos de investigación exploratoria, intervención y extensión que se realizaron estos años fueron bien interesantes y productivos en el sentido de que aportaron desde elementos teóricos y metodológicos no solo al curso en sí sino a la Universidad y a las cooperativas en general. Dentro de los trabajos desarrollados todos estos años, creemos que es necesario hacer mención a los títulos de cada trabajo y con las diversas experiencias asociativas, cooperativas y colectivas en general que han sido llevados a cabo por los estudiantes del curso.

Las áreas seleccionadas por los estudiantes fueron: cárcel, Mesa Intercooperativa, cooperativas sociales, cooperativas de vivienda, cooperativas de trabajo, cooperativas de consumo, sociedad de fomento rural, colectivo de transexuales, colectivos de productores rurales, entre otros. Los trabajos que se mencionan son los efectuados desde 2012 hasta 2016, pues el año 2017 está en curso: - "¿Y si nos asociamos? Un desafío para el trabajo asociativo en contextos de encierro"

- "Participación en la intercooperación: estudio de caso sobre la Mesa Intercooperativa de Salto"

- "Cooperativas sociales, ¿cuánto de proceso motivacional, autonomía y autogestión?"

- "Prácticas Intercooperativa"

- "Transmisión de valores y principios cooperativos en las cooperativas de vivienda"

- "La educación cooperativista"

- "Igualdad de género en el sistema cooperativo: ¿una situación posible?"

- "Cooperativismo de vivienda y discapacidad"

- "Aplicación de valores y principios cooperativos de los hijos de los cooperativistas de vivienda"
- "Dos modelos habitacionales: semejanzas y diferencias"

- "Si de la tierra depende la vida, luchemos por ella..."

- "Salud en la Lucha"

- "Factores que influyen en la participación de las actividades de la Sociedad de Fomento Rural de Colonia 18 de Julio"

- "Federación Uruguaya de Cooperativas de Vivienda por ayuda mutua - FUCVAM y las cooperativas de vivienda: dos realidades distintas"

- "Desde la perspectiva joven: el cooperativismo"

- "Aprendiendo vivencialmente que la esperanza es inseparable de la lucha: cooperativa social en formación"

- "Aprender haciendo... cooperativa de vivienda"

- "Apostando al cambio de la realidad productiva de cooperativa YVY (YVY significa Tierra en Guaranî)"

- "Desigualdad de género en el área rural"

- "Desafíos del género femenino en la actualidad"

- “CCómo influye el desempeño de los diferentes roles en los mecanismos de participación de la cooperativa de vivienda?"

- "Levantar nuestras casas, el punta pié inicial..."

- "Situación de la unión trans del Uruguay, en Salto"

- "Arte por y para la gente"

- "Percepción de valores y principios cooperativos de los jóvenes"

- "Educación y formación cooperativa"

- "Abordaje colectivo entre el grupo Parada Viña (PV) y estudiantes del Curso de Cooperativismo, frente a la inquietud de conformar una cooperativa por parte del grupos Parada Viña"

- "Cooperativismo, una forma de vida..."

- "Incidencia de los cursos de cooperativismo brindados por la

Mesa Intercooperativa de Salto (MIS) y sus efectos"

- "Acercándonos a Cooperativa de Vivienda Azahares, a través del pensamiento de sus miembros".

Ya sean de investigación exploratoria o extensión, se desarrollan bajo el acompañamiento y supervisión de los docentes, y los realizados en el marco de este curso se diferencian de los de otros cursos que componen las carreras de grado de la Universidad por el hecho de que los estudiantes no son solo de la academia sino que también hay profesionales, trabajadores y cooperativistas en general. Lo interesante y productivo del formato pedagógico de este espacio de formación es el desarrollo de prácticas integrales en el área del sector cooperativo-asociativo en general, donde se producen intercambios de saberes individuales y colectivos, uniéndose para la realización de un proyecto, partiendo de una situación problema seleccionada por los propios estudiantes, con una parte de la realidad del sector asociativo-cooperativo, y en procura de construir en este ámbito conocimiento desde las prácticas, desde las experiencias. En este sentido, se apuesta a los aportes efectuados desde los participantes en una situación particular de esa realidad seleccionada. 


\section{Reflexiones desde la actividad docente en torno a prácticas} integrales en cooperativismo

La búsqueda de los docentes es motivar una participación más democrática y asociativa, de manera de promover una lógica más coherente entre la forma y el contenido. La idea de abordar lo "colectivo" requiere un aprendizaje compartido, de compromiso y responsabilidad de todas/os quienes participan, tanto estudiantes como cooperativistas, educadores y docentes. También en esta lógica, cada año en una modalidad colectiva, se convoca a actores del sector asociativo-cooperativo con el propósito de conocer las experiencias e intercambiar interrogantes y conocimientos. La convocatoria a esta presentación está en función a lo que año a año los estudiantes eligen como área de trabajo. El objetivo aquí es que los estudiantes conozcan otras experiencias, además de las elegidas por ellos para el trabajo en grupo, dado a que algunos como futuros profesionales y otros ya profesionales trabajarán en algún momento con alguna experiencia asociativa-cooperativa. $Y$ para eso, desde el curso se ofrecen esos espacios para generar un intercambio de saberes y acerca de qué aportes se pueden hacer a la academia desde los que forman parte de cada edición del curso, estudiantes, cooperativistas y profesionales. Respecto de esto, desde el Centro Universitario de Salto se busca satisfacer esta demanda, se hace énfasis en la continuidad del mismo, se fomenta la reflexión y el conocimiento sobre cooperativismo y asociativismo en el departamento de Salto y se promueve una mayor participación de cooperativas o asociaciones, ya que se cree que desde el curso las experiencias y vivencias de las cooperativas o grupos asociados son un elemento que aporta mucho en contenido y favorece el intercambio de aprendizaje de todos los que formamos parte del curso. Como ya se ha expresado, el trabajo que los estudiantes realizan con el sector asociativo-cooperativo, en cuanto a la formalidad de los proyectos y el tiempo, está dado en el marco de duración del curso.

En este ámbito, la UdelaR tiene como responsabilidad formar profesionales que, antes de ser especializados en determinadas temáticas, es necesario que se desarrollen como personas capaces de escuchar al que está enfrente y de esta forma procurar ser cooperativos con los grupos, organizaciones y colectivos en general, con los cuales se trabaja. Sentir la importancia de construir con el otro y, en esa construcción, tanto los actores universitarios y actores colectivos en general, siempre tienen algo para aportar desde lo académico y más desde la experiencia cotidiana, con el propósito de llegar a la meta propuesta. Y se hace mención al cooperativismo y a la UdelaR porque estudiantes y docentes pensamos que cuando vamos a trabajar con un colectivo está todo diseñado, todo claro y, en este caso, ese colectivo también cree que el que puede hablar, o pensar una solución, es el profesional. Pues no, trabajar en cooperación implica que la Universidad aporte su saber académico en complemento con el saber de la gente con la que se trabaja, porque los profesionales universitarios saben cómo se diseña un proyecto pero la gente con la que se trabaja en territorio aporta el saber de su día a día, en la realidad de sus vidas cotidianas. $Y$ hay que ser capaz de adecuarse, complementarse del modo más asociativo y cooperativo posible.

En este contexto académico, lo que se busca es que, independientemente de lo que dura el curso, es necesario y primordial el vínculo que los estudiantes puedan construir con las cooperativas y colectivos en general. $Y$ en este sentido, se apuesta y se pone el énfasis no solo en el vínculo que se pueda construir más allá del curso mediante los proyectos de investigación, intervención o extensión, sino también en que durante ese proceso de intercambio de experiencias, conocimientos, se produzca un verdadero diálogo de saberes entre universitarios y comunidad. Con referencia al diálogo de saberes, Balbín expresa:

"El diálogo de saberes en educación popular e investigación comunitaria se ha comprendido como principio, enfoque, referente metodológico y como un tipo de acción caracterizada por el reconocimiento de los sujetos participantes en procesos formativos o de construcción grupal de conocimientos". (1986:1)

Es en este espacio de formación donde se promueven las prácticas integrales, donde se apuesta firmemente desde la convicción de la actividad docente $y$, por ende, desde una necesidad institucional académica el desarrollo de las funciones universitarias. Es en este espacio en donde se apuesta a que los docentes, estudiantes, cooperativistas y profesionales, desarrollen un verdadero diálogo de saberes. Este diálogo se considera condición indispensable para que se puedan reconocer los saberes de todos los participantes y los aportes que cada uno puede hacer desde distintos lugares. Reconocer, a su vez, que muchas veces se ignora más de lo que se sabe, y por eso se necesitan saberes construidos con otros. Zambrano (2000), citado por Diego Germán, manifiesta:

"Hay un saber apegado al vivir, a la experiencia, y que tiene que serlo así porque tiene que conectar con la vivencia propia de quien lo comunica, y que tiene que tener la posibilidad de conectar también con la vivencia propia de quien lo escucha". (2016:85)

\section{Reflexiones desde la práctica docente y algunos desafíos} Curricularizar conlleva el desafío de igualar la extensión al nivel de las demás funciones universitarias, lo que exige la renovación de la enseñanza, la reformulación de los planes de estudio y de las prácticas docentes. Esto implica a su vez, considerar la polisemia de la noción de extensión según los marcos de concepción que cada docente tiene y la heterogeneidad de actividades, proyectos 


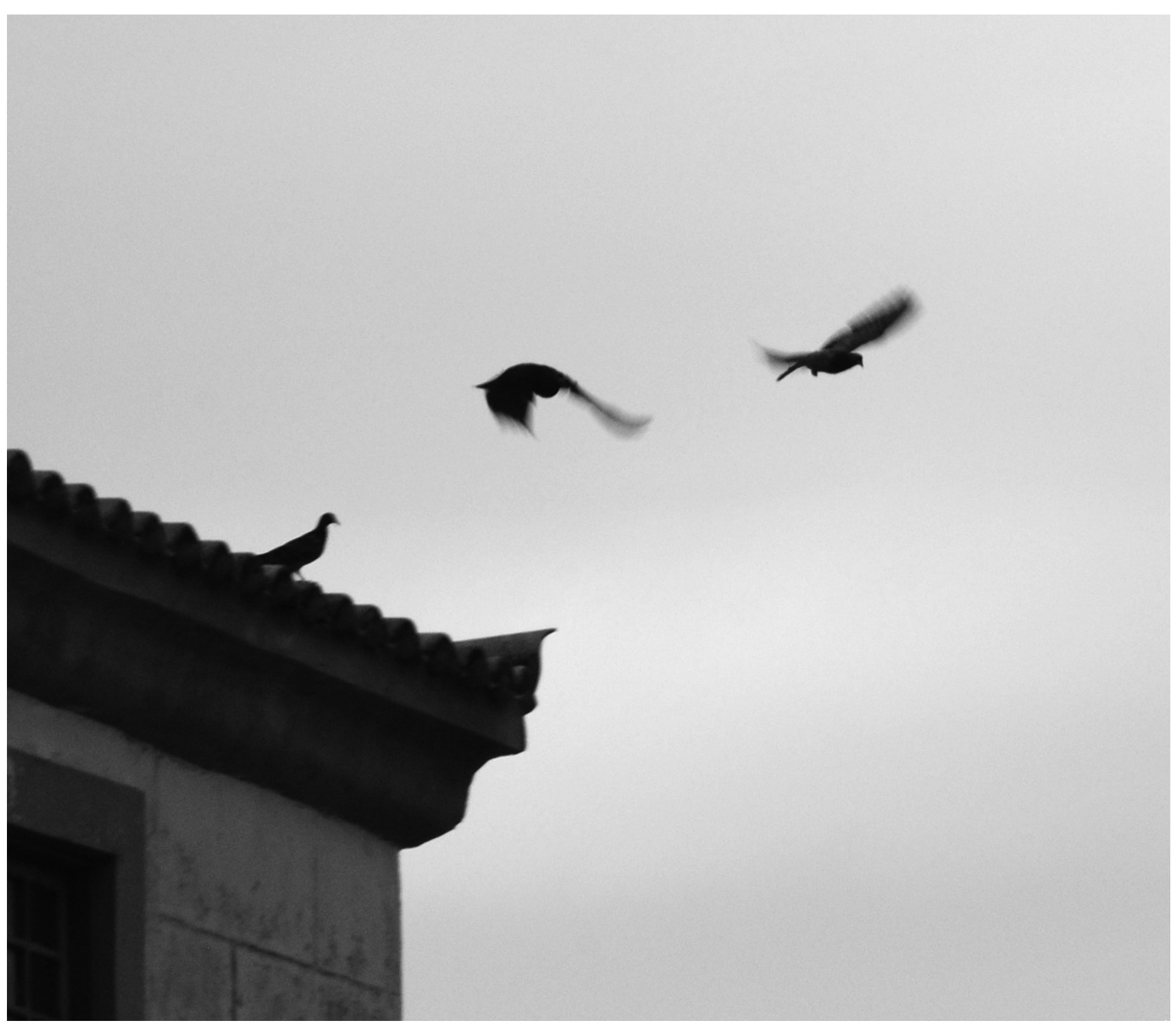

\section{6}

La coordinación docente y de las prácticas en terreno ante la masividad estudiantil, así como la construcción del vínculo con otros actores sociales e institucionales, implica procesos complejos 
y programas que bajo esta función se enmarcan. Las formas de entender y hacer extensión están vinculadas a concepciones de universidad. La yuxtaposición de distintos modelos de universidad coexistentes genera la polisemia en torno a la concepción de extensión. Estos aspectos hacen a una discusión más amplia que involucra el debate político y epistemológico del modelo de universidad que pretendemos.

Gestionar la función de extensión en este marco de curricularización constituye otro engranaje de tipo metodológico. La coordinación docente y de las prácticas en terreno ante la masividad estudiantil, así como la construcción del vínculo con otros actores sociales e institucionales, son algunos de los componentes de la gestión que implica procesos complejos, donde se pone en juego la dimensión ética y se procura evitar formas de invasión cultural.

Las propuestas de formación en extensión en el contexto institucional actual son intervenciones educativas de gran relevancia y necesarias para dar a conocer a estudiantes y docentes el recorrido histórico que la extensión ha tenido en el contexto latinoamericano y nacional y en su proceso de institucionalización. Así como para acercar a los universitarios las orientaciones estratégicas definidas, las distintas concepciones de extensión, el papel de la teoría en los proyectos y los problemas éticos de la intervención en el desarrollo del vínculo universidad-sociedad.

Otro de los desafíos que tenemos como docentes que formamos y nos formamos, a la vez, junto con el resto de los colegas universitarios y con los estudiantes, por supuesto, en el área de la formación en extensión universitaria, es la sistematización de experiencias. En ese encuentro de diálogos de saberes dentro de la Universidad y de esta para con la sociedad, es fundamental la sinergia entre la teoría y la práctica pero, dentro de ello, es necesario saber y entender que la teoría tiene que ser acorde a la realidad, o el recorte de realidad que queremos conocer e intervenir, con el propósito de ejercer una acción transformadora. A propósito de ello, resulta relevante citar a Freire cuando señala: "cuanto más pienso críticamente, rigurosamente, la práctica de la que participo o la práctica de otros, tanto más tengo la posibilidad primero de comprender la razón de ser de la propia práctica, segundo por eso mismo, me voy volviendo capaz de tener una práctica mejor. Así fue, también, como nunca dicotomicé teoría de práctica, jamás las percibí aisladas una de la otra, pero sí en permanente relación contradictoria, procesual”. (1994:125)

\section{Referencias bibliográficas}

Apezteguía, M.; Benelli, L.; Morales, S. (2013). Programa Integral Temático Educación y Comunidad. Sedes Salto y Paysandú, CENUR Litoral Norte, UdelaR. Balbín, J. (1986). Diálogo de saberes; una búsqueda. Cinep, Bogotá. En Ghiso, A., Potenciando la diversidad. Diálogo de saberes, una práctica hermenéutica colectiva. Recuperado de: bibliotecadigital.conevyt.org.mx/colecciones/documentos/ (consultado el 16 de junio de 2017).

Cano, A. (2012). La metodología de taller en los procesos de educación popular. Revista Latinoamericana de Metodología de las Ciencias Sociales, 2(2), 22-51. En Memoria Académica. Disponible en: http://www.memoria.fahce.unlp.edu.ar/ art_revistas/pr.5653/pr.5653.pdf (consultado el 20 de junio de 2017).

Germán, D. (2016). Aportes de la Educación Popular al Cooperativismo. Documento de creación para una clase dentro del Curso de Asociativismo y Cooperativismo. Centro Universitario de Salto. UdelaR.

Freire, P. (1994). Educación en la ciudad. México: Siglo XXI Editorial. Luckesi, C. (1974). Planejamento e Avaliação na Escola: articulação e necessária determinação ideológica. São Paulo: Centro de Referencia en Educación. Recuperado en http://www.crmariocovas.sp.gov.br/dir_a.php?t=015

Najmanovich, D. (1998). Interdisciplina: riesgos y beneficios del arte dialógico. Tramas, (IV), 4. Montevideo: Asociación Uruguaya de Psicoanálisis de las Configuraciones Vinculares.

UdelaR (2005). Plan Estratégico de la Universidad. Montevideo.

(2010). La extensión en la renovación de la enseñanza: Espacios de Formación Integral. Hacia la reforma universitaria, (10). Montevideo.

(2011). Ordenanza de Estudios de Grado y otros Programa de Formación Terciaria. Recuperado de: www.universidad.edu.uy 